\title{
Socio-demographic and structural barriers to being tested for chlamydia in general practice
}

G eneral practice is at the forefront of health care in Australia, with more than $85 \%$ of the Australian population consulting a general practitioner each year. ${ }^{1}$ Preventive health care is an important activity in general practice, and includes the prevention of illness, the early detection of infection or disease, and the promotion and maintenance of health. ${ }^{2}$ While prevention is key to Australia's future health, it is also critical in redressing the health disparities experienced by disadvantaged and vulnerable populations.

Chlamydia screening is a key preventive care activity for young Australian adults. Guidelines recommend that sexually active men and women aged 15-29 years have an annual chlamydia test, ${ }^{2}$ but less than $10 \%$ of this age group are screened each year in general practice. $^{3}$ There are well documented barriers to the uptake of chlamydia screening, from the perspective of both the GP and the patient. For GPs, these barriers include time, lack of awareness or knowledge about chlamydia, lack of support for partner notification, and concern about embarrassing their patient. ${ }^{4,5}$ For patients, barriers include the cost of the GP consultation and the chlamydia test, availability of transport to the clinic, lack of knowledge about the need for testing, the location of the pathology collection site, embarrassment or unease about providing a specimen, and, for those living in rural and remote areas of Australia, fears about confidentiality, the lack of female GPs, and health care availability. ${ }^{6-12}$

The Australian Government funded a chlamydia screening trial in 2009 to determine whether annual chlamydia testing in general practice for 16-29-year-old men and women would reduce the population burden of chlamydia. ${ }^{13}$ As part of this ongoing trial, clinics in the interven- tion group have provided a

\section{Abstract}

Objectives: To investigate socio-demographic and structural factors associated with not providing a specimen for chlamydia testing following a request by a general practitioner.

Design, setting and participants: Cross-sectional analysis of chlamydia testing data for men and women aged 16-29 years attending general practice clinics participating in a cluster randomised controlled trial evaluating the effectiveness of a chlamydia testing intervention. The study period was the 2013 calendar year.

Outcome: The proportion of chlamydia test requests for which the patient did not provide a specimen for testing.

Results: During the study period, there were 13225 chlamydia test requests, for which a chlamydia test was not performed in 2545 instances (19.2\%; 95\% Cl, 16.5-22.3\%). Multivariate analysis indicated that the odds for not undertaking a requested test were higher for men (adjusted odds ratio [aOR], 1.4; 95\% Cl, 1.3-1.6), those aged $16-19$ years (aOR, 1.3; 95\% Cl, 1.1-1.4), those living in areas of greater socio-economic disadvantage (aOR, 1.2; 95\% Cl, 1.1-1.4 for each additional quintile of Index of Relative Socio-economic Disadvantage), and those attending clinics without on-site pathology collection (aOR, 1.4; 95\% Cl, 1.0-1.9).

Conclusion: One in five young people did not submit a specimen for chlamydia testing despite their GP requesting it. This highlights the need for clinics to establish systems which ensure that men and those aged 16-19 years undertake chlamydia tests requested by a GP.

multifaceted intervention designed to facilitate increased chlamydia testing.

We found in an earlier study that not all patients will follow through and have a chlamydia test when a GP requests one. ${ }^{14}$ Our trial provided the opportunity to investigate factors associated with not having a test after it has been requested by a GP. Further, our findings can be extrapolated to other preventive health activities in general practice where tests are requested.

\section{Methods}

\section{Setting}

The Australian Chlamydia Control Effectiveness Pilot (ACCEPt) is a cluster randomised controlled trial of a chlamydia testing intervention in general practice that commenced in mid-2010 and will be completed in late 2015. All 134 GP clinics in 54 rural and regional towns in Victoria (18 towns), New South Wales (21), South Australia (four) and Queensland (11) were recruited for the trial. A further eight clinics in metropolitan Melbourne were also recruited, for a total of 142 clinics. To be eligible for inclusion in the trial, towns had to have a minimum population of 500 people aged 16-29 years (2006 census) and fewer than seven clinics. Towns were excluded if a military base, university or mine was nearby, or if it was a tourist town. A list for each state was drawn up, and towns randomly selected until the required sample size was obtained. More than $90 \%$ of invited clinics agreed to participate. Towns were randomised to either a chlamydia testing intervention group or to a control group. Clinics in the intervention group were asked to target 16-29-year-old men and women for annual testing; they received a multifactorial intervention that included audit and feedback on their chlamydia testing rates, and incentive payments for each 
chlamydia test requested. Control clinics were requested to continue usual chlamydia control practices.

\section{Data collection}

A data extraction tool (GRHANITE) was installed on computers in 122 of the 142 participating clinics. Reasons for not being able to install the tool included the clinic not having a computer (two clinics), non-compatible medical records software (12 clinics) and refusal by the clinic (six clinics). This tool extracts de-identified patient consultation and chlamydia testing data on a weekly basis from the patient medical records database. ${ }^{15}$ Compatible medical records software includes Medical Director, Best Practice, GENIE, Zedmed, Medtech 32, Communicare, SHIP and practiX. Data included in the analysis were patient age and sex, clinic postcode, chlamydia test request (yes v no) and chlamydia test result (positive $\mathrm{V}$ negative $\mathrm{v}$ test not done). Additional clinic-specific data were obtained, such as whether the clinic bulk-billed consultations (a payment option in Australia: the doctor does not charge the patient for the consultation but is instead reimbursed directly by Medicare) and whether pathology or specimen collection was available on site. The socio-economic profile of the geographical location of each clinic was obtained by linking their postcodes with the Australian Bureau of Statistics' 2011 SocioEconomic Indexes for Area-Index of Relative Socio-economic Disadvantage (SEIFA-IRSD). ${ }^{16}$ The IRSD scores areas according to relative disadvantage, ranging from 600 (most disadvantaged) to 1200 (least disadvantaged). The geographical location of the clinic was also classified according to rurality according to the 2007 Australian Standard Geographical Classification SystemRemoteness Area (ASGC-RA). ${ }^{17}$ The ASCG-RA categorises areas according to their physical distance from urban centres (ie, access to goods and services). Its categories, in order of increasing remoteness, are major cities, inner regional, outer regional, remote, and very remote. No participating clinic was in the very remote category. For each clinic postcode, the proportion of the population with Aboriginal and/or Torres Strait Islander background was obtained. Chlamydia prevalence is higher among Aboriginal people, and it is important to determine whether there are any barriers to their having a test when requested by a GP. ${ }^{18}$

\section{Data analysis}

All chlamydia test requests were extracted for the period 1 January 2013 - 31 December 2013. The primary outcome for analysis was defined as "no test conducted" following a GP request. Factors associated with this outcome were explored using logistic regression; univariate and multivariate odds ratios (ORs) and 95\% confidence intervals (CIs) were calculated. All variables were included in the multivariate analysis. Effect modification was explored by fitting interaction terms to the model. Our main analysis was restricted to clinics participating in the intervention arm of the trial because of the availability of data. A total of 63 of 70 intervention clinics provided data for this analysis. We were able to investigate any potential selection bias by comparing the proportions of no test outcomes for intervention and control clinics. All analyses were performed in Stata 13 (StataCorp) and were adjusted for intracluster correlation at the clinic level. IRSD scores were categorised as quintiles for ease of interpretation and analysed as a linear variable, using the least disadvantaged category as reference. Age was categorised in bands (16-19 years, 20-24 years, 25-29 years).

\section{Ethics approval}

ACCEPt received ethics approval from the Royal Australian College of General Practitioners Ethics Committee (NREEC reference number 09/019).

\section{Results}

During the study period, the overall annual testing rate of 16-29-year-old men and women in the 63 intervention clinics was $19.2 \% \quad(95 \% \mathrm{CI}$, $16.8-21.8 \%$ ). A total of 13225 chlamydia tests were requested; 9712
(73\%) were for women. Overall, $29 \%$ of chlamydia test requests were for patients aged 16-19 years; $21 \%$ were requested by clinics in metropolitan Melbourne, $55 \%$ by inner regional clinics, $22 \%$ by outer regional clinics, and $2 \%$ by clinics in remote locations. About one-third of clinics (31\%) provided bulk-billing services for students and young adults; $92 \%$ provided on-site specimen collection for pathology (Box).

No test was conducted for 2545 of the requests $(19.2 \%$; 95\% CI, $16.5-$ $22.3 \%$ ). Univariate analysis indicated that the odds that no test was conducted were higher for men, those aged 16-19 years, and those living in areas of higher socio-economic disadvantage. Multivariate analysis indicated that the odds of no test being conducted were higher for men (adjusted OR [aOR], 1.4; 95\% CI, 1.3-1.6), those aged 16-19 years (aOR, 1.3; 95\% CI, 1.1-1.4), those living in areas of greater socioeconomic disadvantage (aOR, 1.2; 95\% CI, 1.1-1.4 for each additional quintile of Index of Relative Socioeconomic Disadvantage), and those attending clinics without on-site pathology collection (aOR, 1.4; 95\% CI, 1.0-1.9). The geographical location of the clinic, the availability of bulk-billing services, and the proportion of the population who identified as Aboriginal and/or Torres Strait Islander were not associated with the likelihood of a test not being done. No significant interactions between variables were detected.

\section{Assessment of selection bias}

During the study period, the overall annual testing rate in 59 control clinics was $10.9 \%$ (95\% CI, 9.4$12.8 \%$ ). A total of 7148 chlamydia tests were requested; no test was conducted in 1219 instances $(17.1 \%$; $95 \% \mathrm{CI}, 12.9-22.2 \%)$. There was no difference between intervention and control clinics in the proportion of instances where no test was conducted (OR [intervention $v$ control clinics], 1.2; 95\% CI, 0.8-1.7; $P=$ 0.42). As for the intervention clinics, univariate analysis indicated that the odds of a test not being conducted were greater for men (OR, 1.2; 95\% CI, 1.1-1.4) and those aged 
Factors associated with a patient not having a test for chlamydia following a request by a general practitioner

\begin{tabular}{|c|c|c|c|c|c|c|}
\hline Characteristic & $\begin{array}{c}\text { No tests done } \\
\text { (total test requests) }\end{array}$ & $\begin{array}{l}\text { No tests done as } \\
\text { proportion of } \\
\text { test requests } \\
\end{array}$ & $\begin{array}{l}\text { Univariate OR } \\
(95 \% \mathrm{Cl}) \\
\end{array}$ & $P$ & $\begin{array}{l}\text { Multivariate OR } \\
(95 \% \mathrm{Cl}) *\end{array}$ & $P$ \\
\hline Total & 2545 (13255) & $19.3 \%$ & & & & \\
\hline \multicolumn{7}{|l|}{ Sex } \\
\hline Women & $1738(9712)$ & $17.9 \%$ & 1 & & 1 & \\
\hline Men & 816 (3513) & $23.2 \%$ & $1.4(1.2-1.6)$ & $<0.01$ & $1.4(1.3-1.6)$ & $<0.01$ \\
\hline \multicolumn{7}{|l|}{ Age } \\
\hline 16-19 years & 805 (3807) & $21.1 \%$ & $1.2(1.1-1.4)$ & $<0.01$ & $1.3(1.1-1.4)$ & $<0.01$ \\
\hline $20-24$ years & 1072 (5677) & $18.9 \%$ & $1.1(0.9-1.2)$ & 0.30 & $1.1(0.9-1.2)$ & 0.37 \\
\hline $25-29$ years & 668 (3741) & $17.9 \%$ & 1 & & 1 & \\
\hline \multicolumn{7}{|l|}{ Clinic location ${ }^{\dagger}$} \\
\hline Major cities & 559 (2757) & $20.3 \%$ & 1 & & 1 & \\
\hline Inner regional & 1349 (7322) & $18.4 \%$ & $0.9(0.4-1.8)$ & 0.74 & $0.6(0.3-1.3)$ & 0.23 \\
\hline Outer regional & 572 (2871) & $19.9 \%$ & $1.0(0.5-2.0)$ & 0.95 & $0.7(0.3-1.7)$ & 0.47 \\
\hline Remote & 65 (275) & $23.6 \%$ & $1.2(0.5-3.0)$ & 0.67 & $0.8(0.4-1.9)$ & 0.65 \\
\hline $\begin{array}{l}\text { Index of Relative Socio-economic } \\
\text { Disadvantage }{ }^{\ddagger}\end{array}$ & NA & NA & $1.2(1.1-1.3)$ & $<0.01$ & $1.2(1.1-1.4)$ & 0.01 \\
\hline \multicolumn{7}{|l|}{ Bulk-billing } \\
\hline Yes & 1726 (9141) & $18.9 \%$ & 1 & & 1 & \\
\hline No & 819 (4084) & $20.1 \%$ & $0.9(0.6-1.5)$ & 0.76 & $1.2(0.8-1.8)$ & 0.36 \\
\hline \multicolumn{7}{|l|}{ On-site pathology collection } \\
\hline Yes & $2292(1219) 2$ & $18.8 \%$ & 1 & & 1 & \\
\hline No & $253(1033)$ & $24.5 \%$ & $1.4(1.0-2.0)$ & 0.08 & $1.4(1.0-1.9)$ & 0.05 \\
\hline \multicolumn{7}{|l|}{$\begin{array}{l}\text { Aboriginal and/or Torres } \\
\text { Strait Islander }\end{array}$} \\
\hline$<0.6 \%$ & 573 (3415) & $16.8 \%$ & 1 & & 1 & \\
\hline $0.6 \%-1.1 \%$ & $940(4178)$ & $22.5 \%$ & $1.4(0.9-2.2)$ & 0.10 & $1.4(0.8-2.4)$ & 0.24 \\
\hline $1.2 \%-2.5 \%$ & $383(2326)$ & $16.5 \%$ & $0.9(0.7-1.4)$ & 0.91 & $1.1(0.6-1.8)$ & 0.99 \\
\hline$\geq 2.6 \%$ & 649 (3306) & $19.6 \%$ & $1.2(0.9-1.6)$ & 0.19 & $1.1(0.6-1.8)$ & 0.81 \\
\hline
\end{tabular}

NA = not applicable; OR = odds ratio. * Adjusted for all variables in the table. †Australian Standard Geographical Classification System - Remoteness Area. ${ }^{17}$ ¥IRSD score was categorised into quintiles and analysed as a linear variable using the least disadvantaged area as reference. Odds ratio is for the change in the odds of no test being performed for each additional increase in IRSD quintile. § Proportion of population identifying as Aboriginal and/or Torres Strait Islander; based on reference 19.

16-19 years (OR, 1.3; 95\% CI, $1.1-1.5)$.

\section{Discussion}

We found that about one in five young people for whom chlamydia tests were requested by a GP did not submit a specimen. Men, people aged 16-19 years, those living in areas of greater socio-economic disadvantage, and those attending clinics without on-site pathology collection were less likely to follow through and be tested for chlamydia after a GP request. It is important to note that lower age and socio-economic disadvantage are key risk factors for chlamydia, ${ }^{20}$ highlighting how vital it is that clinics establish systems which ensure that tests ordered by GPs are actually undertaken.

We found that men were more likely to not undertake a requested test. Qualitative research has provided some insight into possible reasons for this. An evaluation of a peer-led intervention in the United Kingdom that aimed to increase chlamydia screening found that embarrassment was a key issue deterring young men from screening tests, whereas women were more open and accepting. ${ }^{21}$ Another UK study found that men's attitudes to chlamydia screening were affected by a lack of knowledge and social embarrassment about chlamydia, a reluctance to seek medical help, a perception that chlamydia was a "women's disease", and indifference to health promotion campaigns. ${ }^{22}$

We found that those aged 16-19 years were less likely to undergo a test requested by a GP. It is possible that concern about confidentiality and privacy in general practice may have deterred some from chlamydia screening. ${ }^{10}$ Young people are also less likely to undergo annual health checks or to seek health 
information, $^{23}$ and they express uncertainty or misconceptions about what the test entails. ${ }^{24}$ It has been argued that simply raising awareness about the risk of chlamydia may not increase testing rates, and that providing reassurance of noninfection may be more productive. ${ }^{25}$

We found that the odds of not undertaking a requested test rose with increasing levels of socio-economic disadvantage. This is consistent with an earlier study that found that those living in disadvantaged areas in Australia had lower chlamydia testing rates. $^{26}$ This is a concern because socio-economic disadvantage is a risk factor for chlamydia infection, ${ }^{18}$ so it is vital that barriers to chlamydia testing are minimised in these areas. We found that the availability of bulk-billing for the consultation was not an important deterrent. However, we do not have information about whether the cost of the test was also bulk-billed, and it is possible that lack of knowledge about its cost may have deterred some patients from having the test. Qualitative research is needed to examine this possibility.

We found that the odds of not undertaking a test were $40 \%$ higher in clinics without on-site pathology collection. Chlamydia testing can be stressful for some patients, and any inconvenience, such as having to leave the clinic to attend an off-site pathology collection centre, will deter patients from following through with a test, especially if the testing centre is not nearby. Most pathology providers in Australia offer a courier service; if patients were able to leave self-collected specimens, such as urine or vaginal swabs, at a clinic, these could be collected by the provider, potentially increasing the uptake of testing.

A number of limitations must be considered when interpreting our results. Firstly, clinics were not randomly sampled for participation in the screening trial; as a result, our results may be subject to selection bias. However, this is unlikely to be important, as more than $90 \%$ of clinics agreed to participate, our analysis accounted for intracluster correlation within clinics, and we found no differences in results between intervention and control clinics. Secondly, more than $80 \%$ of our clinics were in rural areas, so that it may not be possible to generalise our results to all Australian clinics. Thirdly, data were unavailable for seven of the intervention clinics $(10 \%)$ because we were unable to install our data extraction tool on a clinic computer. Fourthly, we were unable to assess other barriers to testing, such as the physical layout of the clinic, the location of a toilet, and the availability of local transport. Our analysis was also restricted to summary measures of bulk-billing and pathology collection at the clinic. It is possible that bulk-billing at some clinics may be at the discretion of the doctor, and some doctors may be willing to allow a patient to leave a specimen for collection if on-site pathology services are not available. We were also unable to assess the reason for test requests and whether there was any difference in the failure to test between those who were symptomatic or asymptomatic at the time of the request. Finally, it is possible that there were errors in our data extraction, and that not all chlamydia test requests and results were extracted. However, this problem is unlikely to be significant, because we also collected chlamydia testing data from pathology providers to validate the data extracted for each clinic.

The strengths of our study included its large sample size. Further, more than $90 \%$ of clinics agreed to participate, we adjusted our analysis for intracluster correlation within clinics, and we investigated area-level, clinic-level and patient-level factors. We believe our results can be extrapolated to other preventive health activities targeting this age group in general practice.

\section{Conclusions}

We found that men, those aged 16-19 years, those living in areas of increasing socio-economic disadvantage and those attending clinics without on-site pathology collection services were less likely to be tested for chlamydia following a GP request. Qualitative research is needed to understand why these individuals are not tested. This is important information for health service delivery in general practice, and highlights the need for clinics to develop systems which ensure that men, younger people and those living in socioeconomic disadvantaged areas have a test when requested by their GP.

\section{Competing interests: No relevant disclosures.}

Received 13 Aug 2015, accepted 26 Oct 2015.

(c) 2016 AMPCo Pty Ltd. Produced with Elsevier B.V. All rights reserved. 
1 Britt $\mathrm{H}$, Miller $\mathrm{G}$, Henderson J, et al. General practice activity in Australia 2013-14. (General practice series no. 36). Sydney: Sydney University Press, 2014. http://ses.library.usyd.edu.au/ bitstream/2123/11882/4/9781743324226_ ONLINE.pdf (accessed Nov 2015).

2 Royal Australian College of General Practitioners. Guidelines for preventive activities in general practice. 8th ed. East Melbourne: Royal Australian College of General Practitioners, 2012. http://www.racgp.org.au/download/ Documents/Guidelines/Redbook8/ redbook8.pdf (accessed Nov 2015).

3 Kong FYS, Guy RJ, Hocking JS, et al. Australian general practitioner chlamydia testing rates among young people. Med J Aust 2011; 194: 249-252. https://www.mja.com.au/journal/ 2011/194/5/australian-generalpractitioner-chlamydia-testing-ratesamong-young-people

4 Hocking JS, Parker RM, Pavlin N, et al. What needs to change to increase chlamydia screening in general practice in Australia? The views of general practitioners. BMC Public Health 2008; 8: 425.

5 Bilardi JE, Hopkins CA, Fairley CK, et al. Innovative resources could help improve partner notification for chlamydia in primary care. Sex Transm Dis 2009; 36: 779-783.

6 DeWitt DE. Analysis to synergy: why coordination of rural initiatives at metropolitan universities is important and timely. Aust J Rural Health 2007; 15: 225-226.

7 Pavlin N, Gunn JM, Parker R, et al. Implementing chlamydia screening: what do women think? A systematic review of the literature. BMC Public Health 2006; 6: 221-231.

8 Pavlin N, Parker R, Gunn J, et al. Take the sex out of STI screening! Views of young women on implementing chlamydia screening in general practice. BMC Infect Dis 2008; 8: 62.

9 Ward B, Humphreys J, McGrail M, et al. Which dimensions of access are most important when rural residents decide to visit a general practitioner for non-emergency care? Aust Health Rev 2015; 39: 121-126.
10 Warr D, Hillier L. "That's the problem with living in a small town": privacy and sexual health issues for young rural people. Aust J Rural Health 1997; 5: 132-139.

11 Bryson L, Warner-Smith P. Choice of GP: who do young rural women prefer? Aust J Rural Health 1998; 6: 144-149.

12 Cameron H, Dupal P. Rural pathology under the microscope. Aust J Rural Health 2009; 17: 222-223.

13 Hocking JS, Low N, Guy R, et al. Protocol 12PRT/9010: Australian Chlamydia Control Effectiveness Pilot (ACCEPt): a cluster randomised controlled trial of chlamydia testing in general practice (ACTRN1260000297022). http://www. thelancet.com/protocol-reviews/12 PRT-9010 (accessed Nov 2015).

14 Yeung AH, Temple-Smith M, Fairley CK, et al. Chlamydia prevalence in young attenders of rural and regional primary care services in Australia: a crosssectional survey. Med J Aust 2014; 200 : 170-175. https://www.mja.com.au/ journal/2014/200/3/chlamydiaprevalence-young-attenders-rural-andregional-primary-care-services

15 Boyle D, Kong F. A systematic mechanism for the collection and interpretation of display format pathology test results from Australian primary care records. Electron J Health Inform 2011; 6: el8.

16 Australian Bureau of Statistics. 2033.0.55.001 - Census of Population and Housing: Socio-Economic Indexes for Areas (SEIFA), Australia, 2011 [website]. http://www.abs.gov.au/ AUSSTATS/abs@.nsf/DetailsPage/2033. 0.55.0012011?OpenDocument\#Data (accessed Nov 2015).

17 Australian Bureau of Statistics. 1216.0.15.003 - Australian Standard Geographical Classification (ASGC) Remoteness Area Correspondences, 2006 [website]. http://www.abs.gov. au/ausstats/abs@.nsf/Products/1216. $0.15 .003 \sim 2006 \sim$ Main+Features 2006+RA+from+2006+POA+ Correspondence?OpenDocument\# 221227101023994952 (accessed Apr 2015).
18 Australian Bureau of Statistics. 4102.0 - Australian social trends, Jun 2012. Sexually transmissible infections [website]. http://www.abs. gov.au/AUSSTATS/abs@.nsf/ Lookup/4102.0Main+Features10Jun+2 012\#ATSI (accessed Apr 2015).

19 Australian Bureau of Statistics. 3238.0.55.001 - Estimates of Aboriginal and Torres Strait Islander Australians, June 2011. Aug 2013 [website]. http://www.abs.gov.au/ AUSSTATS/abs@.nsf/DetailsPage/ 3238.0.55.001June\%202011? OpenDocument (accessed Apr 2015).

20 Sheringham J, Mann S, Simms I, et al. It matters what you measure: a systematic literature review examining whether young people in poorer socioeconomic circumstances are more at risk of chlamydia. Sex Transm Infect 2013; 89: 175-180.

21 Loaring J, Oliver I, Campbell R, et al. Could a peer-led intervention increase uptake of chlamydia screening? A proof of principle pilot study. J Fam Plann Reprod Health Care 39: 21-28.

22 Chaudhary R, Heffernan CM, Illsley AL, et al. Opportunistic screening for Chlamydia: a pilot study into male perspectives on provision of Chlamydia screening in a UK university. J Public Health 2008; 30: 466-471.

23 Deeks A, Lombard C, Michelmore J, Teede $H$. The effects of gender and age on health related behaviours. $B M C$ Public Health 2009; 9: 213.

24 Richardson D, Maple K, Perry N, et al. A pilot qualitative analysis of the psychosocial factors which drive young people to decline chlamydia testing in the UK: implications for health promotion and screening. Int J STD AIDS 2010; 21: 187-190.

25 Booth AR, Harris PR, Goyder E, Norman P. Beliefs about chlamydia testing amongst young people living in relatively deprived areas. J Public Health (Oxf) 2013; 35: 213-222.

26 McNamee KM, Fairley CK, Hocking JS. Chlamydia testing and notification in Australia: more money, more tests. Sex Transm Infect 2008; 84: 565-569. 